\title{
Impacto del soporte social sobre la movilidad limitada en personas mayores de comunidades altoandinas peruanas
}

\author{
José F. Parodi' y Fernando M. Runzer-Colmenares ${ }^{2}$
}

Forma de citar

Parodi JF, Runzer-Colmenares FM. Impacto del soporte social sobre la movilidad limitada en personas mayores de comunidades altoandinas peruanas. Rev Panam Salud Publica. 2021;45:e88. https://doi.org/10.26633/RPSP.2021.88

RESUMEN

Objetivo. Determinar si existe relación entre la movilidad física y la falta de soporte social en personas mayores que viven en comunidades altoandinas de Perú.

Métodos. Estudio observacional, analítico y retrospectivo a partir de la base de datos de una investigación previa de corte trasversal con información de 449 personas de 60 años o más participantes en el proyecto ANDES-FRAIL. La variable dependiente fue la movilidad, evaluada según el instrumento Short Physical Performance Battery; la variable independiente fue el soporte social, evaluado mediante un ítem del cuestionario de fragilidad de Edmonton. Como covariables se utilizaron parámetros sociodemográficos, comorbilidades, el número de fármacos de consumo habitual y los puntajes recibidos en varios instrumentos: el índice de Barthel para determinar la funcionalidad en personas mayores, el cuestionario de Yesavage para evaluar la depresión en ancianos, entre otros. Se calcularon las frecuencias y los porcentajes de las variables categóricas. Se elaboró un modelo ajustado con las variables que resultaron estadísticamente significativas en el análisis de regresión logística bifactorial.

Resultados. La frecuencia de movilidad limitada en la población estudiada fue 58,6\% $(n=263)$ y la de inadecuado soporte social fue $58,1 \%(n=261)$. Los participantes con inadecuado soporte social tuvieron una frecuencia de movilidad limitada del 70,7\% $(n=186)$. Según el modelo de regresión ajustado, el soporte social inadecuado incrementaría en 2,5 veces (IC95\%: 1,3-4,5) las probabilidades de tener movilidad limitada, independientemente de las covariables confusoras.

Conclusiones. La falta de apoyo social está asociada con la movilidad limitada de las personas mayores que habitan en comunidades altoandinas peruanas.

Palabras clave Apoyo social; población rural; anciano; fragilidad; envejecimiento saludable; Perú.

La heterogeneidad, la diversidad y la pluriculturalidad de los países de América Latina y el Caribe, así como las inequidades existentes en ellos, afectan a la atención de la salud de sus poblaciones, y de manera muy especial la dirigida a las personas mayores que viven en zonas rurales, ya que - aunque gozan de los mismos derechos - tienen necesidades y expectativas diferentes a las de otros segmentos de la población. Perú no es una excepción y se ha documentado que la esperanza de vida de las personas que habitan en sus zonas rurales sigue siendo menor que la de quienes viven en zonas urbanas (1).

El envejecimiento saludable tiene una relación directa con la habilidad funcional de las personas y significa poder "ser

\footnotetext{
1 Facultad de Medicina Humana, Centro de Investigación del Envejecimiento (CIEN), Universidad de San Martín de Porres, Lima, Perú $\square$ jparodig@usmp.pe
} 
y hacer lo que uno considera valioso". Entre las condiciones requeridas para lograr un envejecimiento saludable están poder satisfacer las necesidades básicas, crear y mantener un círculo de contactos sociales, seguir aprendiendo y conservar la movilidad (2). Las personas mayores de las zonas andinas relacionan el "estar envejecido" con no poder continuar trabajando o no ser tomadas en cuenta en su comunidad (3).

La habilidad funcional depende tanto de la capacidad intrínseca (suma de capacidades físicas y mentales de la persona) como del entorno, así como de sus interacciones. La capacidad intrínseca abarca distintos dominios y subdominios, tales como la movilidad, la vitalidad física, y los estados sensorial, psicológico y cognitivo (4). La Organización Mundial de la Salud ha propuesto el modelo denominado Cuidado Integral para Personas Mayores (ICOPE, por las siglas de Integrated Care for Older People) a fin de diseñar y evaluar intervenciones para las personas mayores que incluyan una valoración social (5). Según el principio de integralidad del ser humano, los dominios evaluados se interrelacionan, ya que la intervención o los problemas en uno de ellos podrían influir en los otros; por ello, esos dominios no deben verse de forma aislada $u$ horizontal.

Teniendo en cuenta lo anterior, el objetivo de la presente investigación fue determinar si existe relación entre la movilidad física y la falta de soporte social en personas mayores que viven en comunidades altoandinas de Perú.

\section{MATERIALES Y MÉTODOS}

El presente trabajo es un estudio observacional, analítico y retrospectivo a partir de la base de datos de una investigación previa de corte trasversal realizada en personas de 60 años o más participantes en el proyecto ANDES-FRAIL $(6,7)$. Este proyecto se desarrolló entre 2013 y 2019 en 12 localidades y distritos altoandinos de Perú, ubicados por encima de los 1500 metros sobre el nivel del mar (msnm): Atipayán, Llupa, Macashca y San Pedro de Chaná, en el departamento de Ancash; La Jalca y Leimebamba, en Amazonas; Pampamarca, en Huánuco; Chacapampa, en Huancayo; Ayahuanco, en Ayacucho; Paucarcolla, en Puno; Vilca, en Huancavelica; y Viñac, en Lima.

Se contó con información de 453 personas mayores residentes en las comunidades estudiadas, reclutadas mediante un muestreo de tipo censal no probabilístico que considera a más del 95\% de las personas de 60 años o más de cada comunidad; se excluyeron las personas que no deseaban participar, las que tenían diagnóstico de demencia y las que cometieron ocho errores o más en el cuestionario de Pfeiffer de tamizaje de trastornos neurocognitivos (ajustado según el nivel educacional). La información provino de encuestas realizadas en la comunidad, en las que se recabaron datos sociodemográficos, así como sobre comorbilidades, síndromes geriátricos y uso de servicios de salud; además, se hicieron mediciones antropométricas (6).

En el estudio original, personal médico previamente capacitado entrevistó a los participantes en sus domicilios acompañados de sus cuidadores o familiares para facilitar el registro de la información en caso de trastornos sensoriales.

La variable dependiente fue la movilidad, evaluada según el instrumento conocido como Short Physical Performance Battery (SPPB), recomendado por la Organización Panamericana de la Salud para evaluar la capacidad locomotriz (8), que ya ha sido empleado en poblaciones altoandinas $(9,10)$. El puntaje del SPPB se define en términos de equilibrio, velocidad de marcha, y capacidad de sentarse y ponerse de pie de una silla, mediante una escala de 12 puntos ( 0 no tiene movilidad; 12 sin problemas de movilidad); se considera como deficiente cualquier valor $\leq 8$.

La variable independiente fue el soporte o apoyo social, evaluado mediante un ítem del cuestionario de fragilidad de Edmonton, que mide la respuesta del entorno social de la persona mayor ante alguna necesidad (11). Como covariables se utilizaron parámetros sociodemográficos (edad, sexo, vivir solo, trabajar actualmente, años desde la jubilación y altitud a la que se ubica la comunidad), el número de comorbilidades (hipertensión, diabetes, enfermedad pulmonar obstructiva crónica, lumbalgia y obesidad) y el número de fármacos de consumo habitual. Se utilizaron también los puntajes obtenidos mediante varios instrumentos: el índice de Barthel para determinar la funcionalidad en personas mayores, el cuestionario de Yesavage para evaluar la depresión en ancianos (versión de 5 ítems), el cuestionario de Pfeiffer para trastornos neurocognitivos y la escala de evaluación socio-familiar de Gijón. Se midió también el alcance funcional en centímetros para evaluar el riesgo de caídas y trastornos del equilibrio (7).

En el análisis estadístico se emplearon las frecuencias y los porcentajes de las variables categóricas. Para las variables numéricas (con distribución normal según la prueba de Shapiro-Wilk) se calcularon las medias y sus desviaciones estándar (DE). Para el análisis bifactorial se empleó la prueba de la ji al cuadrado $\left(X^{2}\right)$ para las variables categóricas y la variable dependiente, y la prueba de la $t$ de Student para las variables numéricas. Mediante el análisis de regresión logística con varianza robusta se construyó un modelo crudo para determinar las razones de disparidad (odds ratio, OR) y los intervalos de confianza de 95\% (IC95\%) entre las covariables y la variable dependiente. En el modelo ajustado se incluyeron las variables que resultaron estadísticamente significativas en el modelo crudo. Para los cálculos se empleó el paquete estadístico Stata para Windows, versión 15.0.

Para calcular la potencia estadística de este análisis secundario, se tomó una frecuencia de bajo soporte social en la población altoandina peruana de $56,9 \%$ y una frecuencia de bajo soporte social en personas mayores con movilidad limitada de 70,7\%, con lo que se obtuvo una potencia de $97,8 \%$ en una muestra de 453 individuos (7). Se excluyó la información de cuatro participantes por falta de referencias sobre movilidad (tres) y soporte social (uno). Finalmente, los datos incluidos correspondieron a 449 participantes.

El estudio original contó con la aprobación del Comité de Ética del Centro Médico Naval del Perú. La información y los datos fueron recolectados por los investigadores y luego codificados para proteger la información y la privacidad de los participantes. La base de datos está disponible en https:/ /osf. io/rsc7q/

\section{RESULTADOS}

En el estudio, predominaron las mujeres $(n=283 ; 63,1 \%)$ y el promedio de edad general fue de 72,9 años $(\mathrm{DE}=6,93)$. De los participantes, $263(58,6 \%)$ tenían movilidad limitada y 261 $(58,1 \%)$ contaban con un inadecuado soporte social; no obstante, aproximadamente la mitad ( $n=240 ; 53,5 \%)$ continuaba trabajando. El promedio del tiempo desde la jubilación fue de 
CUADRO 1. Características de la muestra de personas de 60 años o más de comunidades altoandinas de Perú, 2013-2019 $(n=449)$

\begin{tabular}{lc}
\multicolumn{1}{c}{ Variable } & Medición \\
Edad en años, media (DE) & $72,9(6,93)$ \\
Sexo, $n(\%)$ & \\
Mujer & $283(63,1)$ \\
Hombre & $166(36,9)$ \\
Vive solo/a, $n$ (\%) & \\
No & $352(78,4)$ \\
Sí & $97(21,6)$ \\
Soporte social, $n$ (\%) & \\
Adecuado & $188(41,9)$ \\
Inadecuado & $261(58,1)$ \\
Movilidad, $n$ (\%) & \\
Adecuada & $186(41,4)$ \\
Limitada & $263(58,6)$ \\
Trabaja actualmente, $n$ (\%) & \\
No & $209(46,5)$ \\
Sí & $240(53,5)$ \\
Años desde la jubilación/retiro, media (DE) & $4,9(6,7)$ \\
Número de fármacos de consumo habitual, & $1,1(1,4)$ \\
$\quad$ media (DE) & \\
Puntaje del índice de Barthel, media (DE) & $91,0(10,7)$ \\
Puntaje del cuestionario de Pffeifer, media (DE) & $2,1(2,3)$ \\
Puntaje del cuestionario de Yesavage (5-ítems), & $1,8(1,4)$ \\
media (DE) & \\
Alcance funcional en cm, media (DE) & $19,6(6,8)$ \\
Puntaje de la escala de Gijón, media (DE) & $13,4(3,7)$ \\
Número de comorbilidades, media (DE) & $0,6(0,8)$ \\
Metros sobre el nivel del mar del poblado, \\
$\quad$ media (DE) \\
\hline
\end{tabular}

Fuente: elaboración propia.

Nota: DE: desviación estándar.

4,9 años (DE $=6,7)$; la media del puntaje de la escala de valoración socio-familiar de Gijón fue de 13,4 (DE = 3,7) (cuadro 1). En la investigación primaria, el 81,6\% de los encuestados no había cursado la secundaria completa; el 18,1\% tenía secundaria completa y el $0,3 \%$, educación técnica o superior.

Según los resultados del análisis bifactorial (cuadro 2), de los participantes con inadecuado soporte social, 186 (70,7\%) mostraron tener movilidad limitada, una frecuencia significativamente mayor que sus pares con movilidad adecuada (40,3\%; $p<0,001)$. Más mujeres que hombres presentaron movilidad limitada (190; 72,2\% vs 73; 27,8\%, respectivamente; $p<0,001)$; de estos últimos, solo $59(22,4 \%)$ vivían solos, sin diferencia significativa con respecto a sus pares que vivían acompañados $(204 ; 77,6 \% ; p=0,6)$.

El promedio de años desde la jubilación fue significativamente mayor en los participantes con movilidad limitada $(5,4$ años, $\mathrm{DE}=7,4)$ que en los participantes con movilidad adecuada ( 4,3 años, $\mathrm{DE}=5,4 ; p<0,001)$. Se encontró que el número promedio de medicamentos consumidos fue significativamente mayor en el grupo con movilidad limitada $(1,3, \mathrm{DE}=1,6)$ que en el grupo con movilidad adecuada $(0,6, \mathrm{DE}=0,9 ; p<0,001)$.

Como se observa en el cuadro 2 , los valores obtenidos en la evaluación funcional (índice de Barthel), neurocognitiva (cuestionario de Pfeiffer) y afectiva (cuestionario de tamizaje de depresión geriátrica de Yesavage) en el grupo con movilidad limitada fueron peores que en el grupo con movilidad adecuada $(p<0,001)$.

Asimismo, el puntaje promedio en la escala de evaluación socio-familiar de Gijón fue significativamente menor en los participantes con movilidad limitada $(13,7, \mathrm{DE}=3,9)$ que en los que tuvieron una movilidad adecuada $(12,9, \mathrm{DE}=3,3 ; p=0,03)$. Además, los participantes con movilidad limitada residían en localidades de mayor altitud (media $=3370 \mathrm{msnm}, \mathrm{DE}=395,8$ ) que sus pares con movilidad adecuada (media $=3138,7 \mathrm{msnm}$, $\mathrm{DE}=509,1 ; p<0,001)$ y su promedio de alcance funcional fue menor $(17,9 \mathrm{~cm}, \mathrm{DE}=6,5)$ que el del grupo con movilidad adecuada $(21,8 \mathrm{~cm}, \mathrm{DE}=6,4 ; p<0,001)$ (cuadro 2).

Según el modelo de regresión ajustado para determinar asociaciones entre las variables principales, las personas mayores de comunidades altoandinas con soporte social inadecuado tendrían 2,5 veces más probabilidades de tener movilidad limitada (IC95\%: 1,3-4,5) que sus pares con suficiente soporte social, independientemente del sexo, la edad, la situación laboral, el número de fármacos que consume, el número de comorbilidades, el alcance funcional, la altitud en que residen, los puntajes obtenidos en los cuestionarios de Pffeifer y de Yesavage, el índice de Barthel y la escala de evaluación socio-familiar de Gijón. No obstante, algunas covariables incrementarían las probabilidades de movilidad limitada: puntaje en el cuestionario de Yesavage ( $\mathrm{OR}=1,4$; IC95\%: 1,1-1,8), el número de fármacos que consume $(\mathrm{OR}=1,3$; IC95\%: 1,1-1,6) y la altitud en que vive (OR = 1,1; IC95\%: 1,1-1,2), mientras que el sexo masculino fue considerado como factor protector $(\mathrm{OR}=0,4$; IC95\%: 0,2-0,7) (cuadro 3).

\section{DISCUSIÓN}

Según el modelo crudo, la falta de soporte social eleva en 2,5 veces el riesgo de presentar movilidad limitada, y este riesgo se mantiene en el modelo ajustado; esto respalda la idea de la utilidad de las acciones intersectoriales para mejorar la salud y de que los dominios de las capacidades físicas y mentales no deben abordarse de manera aislada, ya que se interrelacionan unas con otras. Esto es especialmente importante en la población de las comunidades altoandinas que, además de estar disminuyendo con respecto a la urbana, ha envejecido rápidamente, entre otras razones, por la migración de los jóvenes en busca de mejores oportunidades de vida (12).

Aunque las personas mayores del área rural altoandina tienen un mejor desempeño físico que las que viven en zonas rurales a nivel del mar, la esperanza de vida sigue siendo menor en los primeros (10); a esto se suma que el déficit calórico en la ingesta dietética en las personas mayores es menor en las zonas rurales del Perú que en las zonas urbanas y Lima Metropolitana (1). Por lo tanto, las indicaciones frecuentes como "hacer ejercicio" y "comer sano" que se ofrecen en las actividades de promoción y prevención podrían no tener el mismo impacto en estos dos diferentes contextos, en parte por la gran diferencia entre los determinantes sociales de la salud de ambas poblaciones (educación, servicios básicos, trasporte, pobreza, etc.)

El modelo de salud centrado en la persona, en potenciar sus capacidades y en optimizar el entorno contribuye a poner fin a la era de la salud basada en la enfermedad (13). Si bien la enfermedad modula el proceso de envejecimiento, de aumento de 
CUADRO 2. Relación entre el soporte social, la movilidad y las demás covariables del estudio, según los resultados del análisis bifactorial $(n=449)$

\begin{tabular}{|c|c|c|c|}
\hline \multirow[t]{2}{*}{ Variable } & \multicolumn{2}{|c|}{ Movilidad } & \multirow[t]{2}{*}{$p$} \\
\hline & Adecuada & Limitada & \\
\hline Edad en años, media (DE) & $71,9(6,4)$ & $73,8(7,1)$ & $<0,001^{a}$ \\
\hline \multicolumn{4}{|l|}{ Sexo, $n(\%)$} \\
\hline Mujer & $93(50,0)$ & $190(72,2)$ & \multirow[t]{2}{*}{$<0,001^{\mathrm{b}}$} \\
\hline Hombre & $93(50,0)$ & $73(27,8)$ & \\
\hline \multicolumn{4}{|l|}{ Vive solo/a, $n(\%)$} \\
\hline No & $148(79,6)$ & $204(77,6)$ & \multirow[t]{2}{*}{$0,600^{\mathrm{b}}$} \\
\hline Sí & $38(20,4)$ & $59(22,4)$ & \\
\hline \multicolumn{4}{|l|}{ Soporte social, $n(\%)$} \\
\hline Adecuado & $111(59,7)$ & $77(29,3)$ & \multirow[t]{2}{*}{$<0,001^{b}$} \\
\hline Inadecuado & $75(40,3)$ & $186(70,7)$ & \\
\hline \multicolumn{4}{|l|}{ Trabaja actualmente, $n(\%)$} \\
\hline No & $70(37,6)$ & $139(52,9)$ & \multirow[t]{2}{*}{$0,001^{b}$} \\
\hline Sí & $116(62,4)$ & $124(47,1)$ & \\
\hline Años desde la jubilación/retiro, media (DE) & $4,3(5,4)$ & $5,4(7,4)$ & $<0,001^{\mathrm{a}}$ \\
\hline Número de fármacos de consumo habitual, media (DE) & $0,6(0,9)$ & $1,3(1,6)$ & $<0,001^{\mathrm{a}}$ \\
\hline Puntaje del índice de Barthel, media (DE) & $95,3(7,5)$ & $87,9(11,5)$ & $<0,001^{\mathrm{a}}$ \\
\hline Puntaje del cuestionario de Pffeifer, media (DE) & $1,3(2,4)$ & $2,5(2,1)$ & $<0,001^{\mathrm{a}}$ \\
\hline Puntaje del cuestionario de Yesavage (5-ítems), media (DE) & $1,2(1,2)$ & $2,1(1,4)$ & $<0,001^{\mathrm{a}}$ \\
\hline Alcance funcional en cm, media (DE) & $21,8(6,4)$ & $17,9(6,5)$ & $<0,001^{\mathrm{a}}$ \\
\hline Puntaje de la escala de Gijón, media (DE) & $12,9(3,3)$ & $13,7(3,9)$ & $0,030^{\mathrm{a}}$ \\
\hline Número de comorbilidades, media (DE) & $0,5(0,6)$ & $0,7(0,9)$ & $0,080^{\mathrm{a}}$ \\
\hline Metros sobre el nivel del mar del poblado, media (DE) & $3138,7(509,1)$ & $3370,0(395,8)$ & $<0,001^{\mathrm{a}}$ \\
\hline
\end{tabular}

CUADRO 3. Análisis de regresión logística para determinar la asociación entre el soporte social, la movilidad limitada y las demás covariables del estudio $(n=449)$

\section{Variables}

Edad en años, media (DE)

Sexo, $n(\%)$

Mujer

Hombre

Vive solo/a, $n(\%)$

No

Sí

Soporte social, $n(\%)$

Adecuado

Inadecuado

Trabaja actualmente, $n(\%)$

No

Sí

Años desde la jubilación/retiro, media (DE)

Número de fármacos de consumo habitual, media (DE)

Puntaje del índice de Barthel, media (DE)

Puntaje del cuestionario de Pffeifer, media (DE)

Puntaje del cuestionario de Yesavage (5-ítems), media (DE)

Alcance funcional en $\mathrm{cm}$, media (DE)

Puntaje de la escala de Gijón, media (DE)

Número de comorbilidades, media (DE)

Metros sobre el nivel del mar del poblado, media (DE)

\section{Modelo crudo, OR (IC95\%) Modelo ajustado, OR (IC95\%)}

$1,1(1,0-1,1) \quad 1,1(0,9-1,1)$

Referencia Referencia

$0,4(0,2-0,5) \quad 0,4(0,2-0,7)$

Referencia NA

$1,1(0,7-1,7) \quad N A$

Referencia Referencia

$3,6(2,4-5,3) \quad 2,5(1,4-4,5)$

$\begin{array}{cc}\text { Referencia } & \text { Referencia } \\ 0,5(0,3-0,7) & 0,6(0,4-1,1) \\ 1,0(1,0-1,1) & 1,0(0,9-1,1) \\ 1,6(1,3-1,9) & 1,3(1,1-1,6) \\ 0,9(0,8-0,9) & 0,9(0,9-1,0) \\ 1,4(1,1-1,7) & 1,2(0,8-1,7) \\ 1,5(1,3-1,8) & 1,4(1,1-1,8) \\ 0,9(0,8-0,9) & 0,9(0,9-1,0) \\ 1,1(1,0-1,1) & 1,0(0,9-1,9) \\ 1,2(1,1-1,5) & 1,3(0,9-1,9) \\ 1,0(1,0-1,0) & 1,1(1,1-1,2)\end{array}$

Fuente: elaboración propia.
Nota: DE: desviación estándar; OR: razón de posibilidades (según el término en inglés odds ratio); IC95\%: intervalo de confianza de 95\%; NA: no aplica. 
la fragilidad y de disminución de la capacidad intrínseca (14), el nuevo modelo propone que la persona "pueda seguir sintiéndose saludable a pesar de vivir con una afección crónica de salud", mientras pueda seguir siendo y haciendo lo que considera valioso. A partir de este concepto, el estado de salud de una persona depende más de la capacidad funcional que de tener o no un diagnóstico específico. Por ejemplo, algunas personas mayores diagnosticadas de artrosis o enfermedad isquémica cerebral con secuelas son independientes, mientras que otras, con las mismas enfermedades, son dependientes. Por lo tanto, en geriatría es más importante gestionar las capacidades y desempeños que acumular diagnósticos de enfermedades. El gran reto de la medicina geriátrica es saber en qué medida y en cuánto tiempo se puede optimizar el desempeño al intervenir en un diagnóstico o biomarcador.

Los conceptos de fragilidad y capacidad intrínseca difieren, pero se relacionan con la dinámica del envejecimiento saludable (15). La fragilidad se refiere a disfunciones multisistémicas (homeoestenosis) que llevan a disfunciones orgánicas, mientras que la capacidad intrínseca se relaciona con las reservas físicas y mentales. Es decir, son "caras diferentes de la misma moneda": una se centra en lo que falta y la otra se enfoca en las reservas y capacidades que quedan. De hecho, el SPPB se usa tanto para medir la fragilidad como la movilidad (uno de los dominios de la capacidad intrínseca), constructos que en su interacción con el entorno se expresan como resiliencia (16). Al respecto, algunos autores resaltan el concepto de fragilidad social (17), que aunque aceptan que se relaciona con la fragilidad física- se asocia más con el funcionamiento físico, la cognición y la depresión, además de ser un posible predictor de la mortalidad (18).

Respecto a las particularidades de la población estudiada, se ha demostrado que existe asociación entre la fragilidad y la calidad de vida en zonas rurales (19), por lo que las intervenciones destinadas a mejorar la calidad de vida de las personas mayores consideradas frágiles deberían abordar también el componente social (19), ya que este es importante para el bienestar psicológico en la vejez. Entre las dimensiones del soporte social, se ha comprobado que el apoyo emocional percibido tiene un efecto protector. Esta relación entre el apoyo social y el bienestar psicológico se debe tomar en cuenta para las intervenciones psicosociales integrales que promueven la salud mental en la vejez (20).

Los resultados presentados aquí confirman datos de la literatura en el sentido de que el enfoque de gestión de la capacidad intrínseca debe evaluarse holísticamente y considerar las interrelaciones entre sus dominios, por ejemplo, la relación de la discapacidad visual con el estado socioeconómico, la velocidad de marcha y la fuerza de prensión $(21,22)$ con la función cognitiva, y las discapacidades visual (23) y auditiva (24) con el estado cognitivo. También se suman la relación existente entre las vulnerabilidades sociales (como la falta de apoyo social), y la cognición y el bienestar psicológico (20). No obstante, se debe tener en cuenta que existen diferencias culturales en la percepción del apoyo social y en su efecto sobre la salud mental (25), por lo que es importante considerar los diferentes entornos incluso dentro de un mismo país o región.

La evaluación de la movilidad, como subdominio de la capacidad intrínseca o criterio clave para evaluar la fragilidad, es un componente fundamental de la evaluación de la persona mayor, tanto en el ámbito clínico como académico. Actualmente, entre los instrumentos más utilizados para medir la fragilidad están la prueba de velocidad de la marcha habitual y la batería de rendimiento físico corto (que incluye a la primera); ambas han demostrado tener capacidad predictiva de eventos relacionados con la salud (hospitalización, caídas, institucionalización, discapacidad y mortalidad). Además, esos indicadores han probado su asociación con condiciones subclínicas y clínicas indirectamente relacionadas con la función física, por ejemplo, la inflamación, el estrés oxidativo y la mortalidad general (26). Si bien la cultura y otros factores contextuales tendrían un impacto significativo en la evaluación de medidas del desempeño físico, estas no pierden su conexión con los desenlaces relacionados con la salud. Por este motivo, el uso de determinantes sociales puede servir para afinar la valoración de las necesidades y expectativas de salud de las personas mayores (27).

Las relaciones entre dominios de la capacidad intrínseca $-\mathrm{O}$, visto de otra forma, de los determinantes multidimensionales de la fragilidad estudiados aquí- han sido descritas en la literatura. En China, un estudio realizado en zonas rurales informó que un bajo autocuidado se asociaba con un escaso apoyo social, y que las mujeres tuvieron más posibilidades de mostrar fragilidad, lo que se alinea con la relación encontrada en el presente estudio entre desempeño físico y soporte social (28). Otra investigación realizada en personas mayores de Malasia mostró que ser frágil, según el fenotipo de Fried, se asoció significativamente con la edad avanzada, el ser mujer, un mayor número de enfermedades crónicas, una función cognitiva deficiente y un nivel socioeconómico bajo (29). En Corea del Sur, un estudio de envejecimiento realizado en el área rural de Pyeongchang indicó que la fragilidad social aumentó el riesgo de discapacidad ante las actividades de la vida diaria y que la evaluación de la fragilidad social es importante, ya que permitió identificar a las personas mayores frágiles a partir de parámetros no físicos. Además, la evaluación de la fragilidad social y física podría facilitar la detección de la discapacidad y los síndromes geriátricos (30). Un estudio prospectivo realizado en México demostró que la discapacidad para realizar las actividades básicas de la vida diaria y el analfabetismo aumentaron la probabilidad de ser clasificado como prefrágil o frágil, y concluyó que la vulnerabilidad social de las personas mayores que viven en zonas rurales está asociada con el estado de fragilidad (31).

En Colombia, los factores asociados significativamente con la fragilidad fueron la edad avanzada, ser mujer, vivir en áreas rurales, tener bajo nivel educacional, un mayor número de enfermedades, insuficientes ingresos corrientes y una mala situación económica en la infancia (32). Al respecto, las enfermedades crónicas modulan el proceso de envejecimiento y aumentan la fragilidad, mediante mecanismos como la inflamación crónica. Si bien hay factores de riesgo globales (hipertensión y educación), otros - como la contaminación del aire en el hogar y la mala alimentación - pueden variar según el nivel de desarrollo económico de un país. Las políticas de salud de las naciones pluriculturales y con inequidades internas deben considerar los factores de riesgo de mayor importancia en los grupos específicos de cada país (33).

Aunque existen antecedentes sobre la relación entre el soporte social y la movilidad en las personas mayores, la geografía, el entorno social y étnico y, sobre todo, el acceso que la población tiene a los servicios de salud hacen imprescindibles datos como los mostrados aquí para tomar decisiones informadas y ajustadas al tipo de población y sus necesidades (34).

Esta investigación tiene algunas limitaciones. En primer lugar, los datos se obtuvieron mediante un muestreo censal 
no probabilístico y las comunidades fueron escogidas por conveniencia de los investigadores, lo que sumado al diseño transversal, impide establecer una relación causal entre la falta de soporte social y la movilidad limitada. No obstante, la información obtenida aclara un problema de salud que requiere planteamientos sanitarios a nivel de curso de la vida y muestra la urgencia de optimizar el entorno (físico y social) y mejorar el acceso a servicios de salud adecuados a las particularidades y expectativas de grupos específicos de personas mayores.

A pesar de las limitaciones señaladas, se puede concluir que la falta de apoyo social está relacionada con la movilidad limitada de las personas mayores que viven en las comunidades peruanas altoandinas estudiadas. Por lo tanto, la acción intersectorial y el manejo de los determinantes sociales de esta región deben tomarse en cuenta en las intervenciones para mejorar la salud y la funcionalidad de las personas mayores.
Contribución de los autores. Los autores JFP (https://orcid. org/0000-0002-0336-0584) y FMRC (http://orcid.org/00000003-4045-0260) concibieron el estudio original, recolectaron y analizaron los datos, interpretaron los resultados, escribieron y revisaron el manuscrito, y aprobaron la versión final.

\section{Conflictos de intereses. Ninguno.}

Agradecimientos. Agradecemos el apoyo logístico de la Facultad de Medicina Humana de la Universidad de San Martín de Porres, Lima, Perú.

Declaración. Las opiniones expresadas en este manuscrito son responsabilidad de los autores y no reflejan necesariamente los criterios ni la política de la Revista Panamericana de Salud Pública / Pan American Journal of Public Health y/o de la Organización Panamericana de la Salud.

\section{REFERENCIAS}

1. Instituto Nacional de Estadística e Informática. Situación de la población adulta mayor: enero-febrero-marzo 2019 [Internet]. Lima: INEI; 2019 [citado el 27 de agosto del 2019]. Disponible en: https:// www.inei.gob.pe/media/MenuRecursivo/boletines/adultomayorjunio.pdf

2. Organización Mundial de la Salud. Informe Mundial sobre Envejecimiento y Salud [Internet]. Ginebra: OMS: 2015 [citado el 27 de agosto del 2019]. Disponible en: https://www.who.int/ageing/ publications/world-report-2015/es /

3. Waters WF, Gallegos CA. Salud y bienestar del adulto mayor indígena [Internet]; 2012 [citado el 2 de abril del 2021. Disponible en: https:/ / www1.paho.org/hq/dmdocuments/gdr-salud-y-bienestar -adulto-mayor-indigena.pdf

4. Cesari M. Biological theory for the construct of intrinsic capacity to be used in clinical settings [Internet]. Geneva: WHO; 2016 [citado el 19 de agosto del 2019]. Disponible en: https:/ /www.who.int/ageing/ health-systems/3_Biological-theory-intrinsic-capacity.pdf

5. World Health Organization. Integrated care for older people (ICOPE): Guidance for person-centred assessment and pathways in primary care. Geneva: WHO [Internet] [citado el 7 de septiembre del 2019]. Disponible en https://www.who.int/ageing/publications/ icope-handbook/en/

6. Meucci RD, Runzer-Colmenares FM, Parodi JF, de Mola CL. Falls among the elderly in Peruvian Andean communities and the rural far south of Brazil: Prevalence and associated factors. J Community Health. 2020;45(2):363-9. doi: 10.1007/s10900-019-00751-5

7. Urrunaga-Pastor D, Moncada-Mapelli E, Runzer-Colmenares FM, Bailon-Valdez Z, Samper-Ternent R, Rodríguez-Mañas L, et al. Factors associated with poor balance ability in older adults of nine high-altitude communities. Arch Gerontol Geriatr. 2018;77:108-14. doi: 10.1016/j.archger.2018.04.013

8. Tavassoli N, Piau A, Berbon C, de Kerimel J, Lafont C, de Souto Barreto $\mathrm{P}$, et al. Framework implementation of the INSPIRE ICOPE-CARE Program in collaboration with the World Health Organization (WHO) in the Occitania region. J Frailty Aging. 2020;10(2):103-9. doi: 10.14283/jfa.2020.26

9. Estela-Ayamamani D, Espinoza-Figueroa J, Columbus-Morales M, Runzer-Colmenares F. Rendimiento físico en adultos mayores de una comunidad rural altoandina peruana. Rev Peru Med Exp Salud Publica. 2013;30(2):358-60. doi: 10.17843/rpmesp.2013.302. 226

10. Estela-Ayamamani D, Espinoza-Figueroa J, Columbus-Morales M, Runzer-Colmenares F, Parodi JF, Mayta-Tristán P. Rendimiento físico de adultos mayores residentes en zonas rurales a nivel del mar y a gran altitud en Perú. Rev Esp Geriatr Gerontol. 2015;50(2):56-61. doi: 10.1016/j.regg.2014.11.001

11. Sáenz-Miguel S, Runzer-Colmenares FM, Parodi JF. Trastornos depresivos en adultos mayores de once comunidades altoandinas del Perú, 2013-2017. Acta Med Peru. 2019;36(1):26-31. doi: 10.35663/ amp.2019.361.632

12. Instituto Nacional de Estadística e Informática. Perú: perfil sociodemográfico, 2017 [Internet]. Lima: INEI; 2018 [citado el 2 de abril del 2021]. Disponible en: https://www.inei.gob.pe/media/ MenuRecursivo/publicaciones_digitales/Est/Lib1539/

13. Cesari M, Marzetti E, Thiem U, Pérez-Zepeda MU, Van Kan GA, Landi F, et al. The geriatric management of frailty as paradigm of the end of the disease era. Eur J Intern Med. 2016;31:11-4. doi: 10.1016/j.ejim.2016.03.005

14. Armstrong NM, Carlson MC, Schrack J, Xue QL, Carnethon MR, Rosano C, et al. Late-life depressive symptoms as partial mediators in the associations between subclinical cardiovascular disease with onset of mild cognitive impairment and dementia. Am J Geriatr Psychiatry. 2018;26(5):559-68. doi: 10.1016/j.jagp.2017.11.004

15. Belloni G, Cesari M. Frailty and intrinsic capacity: Two distinct but related constructs. Front Med (Lausanne). 2019;6:133. doi: 10.3389/ fmed.2019.00133

16. Woo J. Frailty, successful aging, resilience, and intrinsic capacity: A cross-disciplinary discourse of the aging process. Curr Geriatr Rep. 2019;8(2):67-71. doi: 10.1007/s13670-019-0276-2

17. Freer K, Wallington SL. Social frailty: The importance of social and environmental factors in predicting frailty in older adults. Br J Community Nurs. 2019;24(10):486-92. doi: 10.12968/bjen.2019.24.10.486

18. Ma L, Sun F, Tang Z. Social frailty is associated with physical functioning, cognition, and depression, and predicts mortality. J Nutr Health Aging. 2018;22(8):989-95. doi: 10.1007/s12603-018-1054-0

19. Siriwardhana DD, Weerasinghe MC, Rait G, Scholes S, Walters KR. The association between frailty and quality of life among rural community-dwelling older adults in Kegalle district of Sri Lanka: A cross-sectional study. Qual Life Res. 2019;28(8):2057-68. doi: 10.1007/s11136-019-02137-5

20. Nicolini P, Abbate C, Inglese S, Rossi PD, Mari D, Cesari M. Different dimensions of social support differentially predict psychological well-being in late life: Opposite effects of perceived emotional support and marital status on symptoms of anxiety and of depression in older outpatients in Italy. Psychogeriatrics. 2020;21(1):42-53. doi: $10.1111 /$ psyg.12633 
21. Parodi JF, Nieto-Gutiérrez W, Téllez WA, Ventocilla-Gonzales I, Runzer-Colmenares FM, Taype-Rondan A. Velocidad de marcha y desarrollo de trastornos neurocognitivos en adultos mayores: resultados de una cohorte peruana. Rev Esp Geriatr Gerontol. 2018;53(2):73-6. doi: 10.1016/j.regg.2017.08.001

22. McGrath R, Cawthon PM, Cesari M, al Snih S, Clark BC. Handgrip strength asymmetry and weakness are associated with lower cognitive function: A panel study. J Am Geriatr Soc. 2020;68(9):2051-8. doi: 10.1111 /jgs.16556

23. Tran EM, Stefanick ML, Henderson VW, Rapp SR, Chen J-C, Armstrong NM, et al. Association of visual impairment with risk of incident dementia in a women's health initiative population. JAMA Ophthalmol. 2020;138(6):624-33. doi: 10.1001/ jamaophthalmol.2020.0959

24. Armstrong NM, An Y, Doshi J, Erus G, Ferrucci L, Davatzikos C, et al. Association of midlife hearing impairment with late-life temporal lobe volume loss. JAMA Otolaryngol Head Neck Surg. 2019;145(9):794. doi: 10.1001/jamaoto.2019.1610

25. Hoogendijk EO, Suanet B, Dent E, Deeg DJH, Aartsen MJ. Adverse effects of frailty on social functioning in older adults: Results from the Longitudinal Aging Study Amsterdam. Maturitas. 2016;83:45-50. doi: 10.1016/j.maturitas.2015.09.002

26. Patrizio E, Calvani R, Marzetti E, Cesari M. Physical functional assessment in older adults. J Frailty Aging. 2021;10(2):141-9. doi: $10.14283 /$ jfa.2020.61

27. Llibre Rodríguez JJ, Prina AM, Acosta D, Guerra M, Huang Y, Jacob KS, et al. The prevalence and correlates of frailty in urban and rural populations in Latin America, China, and India: A 10/66 population-based survey. J Am Med Dir Assoc. 2018;19(4):287-95.e4. doi: 10.1016/j.jamda.2017.09.026

28. Li J, Zhao D, Dong B, Yu D, Ren Q, Chen J, et al. Frailty index and its associations with self-neglect, social support and sociodemographic characteristics among older adults in rural China. Geriatr Gerontol Int. 2018;18(7):987-96. doi: 10.1111/ggi.13280
29. Ahmad NS, Hairi NN, Said MA, Kamaruzzaman SB, Choo WY Hairi F, et al. Prevalence, transitions and factors predicting transition between frailty states among rural community-dwelling older adults in Malaysia. PLoS One. 2018;13(11):e0206445. doi: 10.1371/ journal.pone.0206445

30. Park H, Jang I-Y, Lee HY, Jung H-W, Lee E, Kim DH. Screening value of social frailty and its association with physical frailty and disability in community-dwelling older Koreans: Aging study of Pyeongchang rural area. Int J Environ Res Public Health. 2019;16(16):2809. doi: $10.3390 /$ ijerph16162809

31. Manrique-Espinoza B, Salinas-Rodríguez A, Salgado de Snyder N Moreno-Tamayo K, Gutiérrez-Robledo LM, Avila-Funes JA. Frailty and social vulnerability in Mexican deprived and rural settings. J Aging Health. 2016;28(4):740-52. doi: 10.1177/0898264315609909

32. Ocampo-Chaparro JM, Reyes-Ortiz CA, Castro-Flórez X, Gómez F. Frailty in older adults and their association with social determinants of health: The SABE Colombia study. Colomb Med (Cali). 2019;50(2):89-101. doi: 10.25100/cm.v50i2.4121

33. Yusuf S, Joseph P, Rangarajan S, Islam S, Mente A, Hystad P, et al. Modifiable risk factors, cardiovascular disease, and mortality in 155722 individuals from 21 high-income, middle-income, and low-income countries (PURE): A prospective cohort study. Lancet. 2020;395(10226):795-808. doi: 10.1016/S0140-6736(19)32008-2 34. Menec V, Bell S, Novek S, Minnigaleeva GA, Morales E, Ouma T, et al. Making rural and remote communities more age-friendly: Experts' perspectives on issues, challenges, and priorities. J Aging Soc Policy. 2015;27(2):173-91. doi: 10.1080/08959420.2014.995044

Manuscrito recibido el 30 de noviembre del 2020. Aceptado para publicación, tras revisión, el 16 de abril del 2021. 


\section{Impact of social support on limited mobility in older people in high Andean communities in Peru}

ABSTRACT Objective. To determine whether there is a relationship between physical mobility and lack of social support in elderly people living in high Andean communities in Peru.

Methodology. Observational, analytical, retrospective study using the database of a previous cross-sectional study containing information on 449 people aged 60 years and over, who participated in the ANDES-FRAIL project. The dependent variable was mobility, evaluated using the Short Physical Performance Battery; the independent variable was social support, evaluated using an item from the Edmonton Frail Scale. Sociodemographic parameters, comorbidities, number of drugs commonly used, and scores received from several instruments were used as covariates. These included the Barthel Index to determine functionality in older people and the Yesavage questionnaire to evaluate depression in the elderly. Frequencies and percentages were calculated for the categorical variables. An adjusted model was developed using the variables that were statistically significant in the two-factor logistic regression analysis.

Results. The frequency of limited mobility in the studied population was $58.6 \%(n=263)$ and the frequency of inadequate social support was $58.1 \%(n=261) ; 70.7 \%$ of participants $(n=186)$ with inadequate social support had limited mobility. According to the adjusted regression model, inadequate social support would make it 2.5 times more likely (95\% Cl: 1.3-4.5) to have limited mobility, regardless of the confounding covariates.

Conclusions. Lack of social support is associated with limited mobility in older people living in high Andean communities in Peru.

Keywords $\quad$ Social support; rural population; aged; frailty; healthy aging; Peru.

\section{Impacto do apoio social na mobilidade reduzida em idosos de comunidades do altiplano andino no Peru}

RESUMO

Objetivo. Determinar se existe relação entre mobilidade física e falta de apoio social em idosos que vivem em comunidades do altiplano andino no Peru.

Métodos. Estudo observacional analítico de coorte retrospectiva baseado em dados obtidos em uma pesquisa transversal anterior com 449 participantes, com idade de 60 anos ou mais, como parte do Projeto ANDES-FRAIL. A variável dependente foi a mobilidade, avaliada com o Short Physical Performance Battery, um instrumento que avalia o desempenho físico funcional com uma série curta de testes. A variável independente foi o apoio social, avaliado por um item da Escala de Fragilidade de Edmonton (The Edmonton Frail Scale, EFS). As covariáveis foram parâmetros sociodemográficos, presença de comorbidades, número de medicamentos de uso habitual e pontuação em vários instrumentos de avaliação, como o índice de Barthel de avaliação da independência funcional de idosos e a Escala de Depressão Geriátrica de Yesavage. Foram calculadas as frequências e as porcentagens das variáveis categóricas e elaborado um modelo ajustado com as variáveis com significância estatística na análise de regressão logística bifatorial.

Resultados. Na amostra estudada, 58,6\% $(n=263)$ apresentavam mobilidade reduzida e 58,1\% $(n=261)$ recebiam apoio social inadequado. Entre as pessoas com apoio social inadequado, 70,7\% $(n=186)$ apresentavam mobilidade reduzida. De acordo com o modelo de regressão logística ajustado, a chance de ter mobilidade reduzida foi 2,5 vezes maior (IC 95\% 1,3-4,5) quando o apoio social era inadequado, independentemente das covariáveis de confusão.

Conclusão. A falta de apoio social está associada à mobilidade reduzida em idosos que vivem em comunidades do altiplano andino no Peru.

Palavras-chave Apoio social; população rural; idoso; fragilidade; envelhecimento saudável; Peru. 